\title{
DOCUMENTOS
}

\section{CONGRESO NACIONAL DE INVESTIGADORES SOCIALES $Y$ MEDICO-SOCIALES SOBRE LA JUVENTUD CHILENA}

\section{CONCLUSIONES GENERALES}

1. Desarrollar un Proyecto entre las Facultades de Medicina y de Filosofía, Humanidades y Educación, por una parte y entre los Ministerios de Educación, y Salud, Justicia y del Interior, por otra, a fin de crear un Comité Permanente orientado hacia:

a) Incentivar y promover la investigación sobre la juventud.

b) Creación de un banco de datos sobre estudios de Juventud, tanto a nivel nacional como internacional. Para este efecto, dicho banco deberá estar conectado a la red internacional.

c) Incentivar la ejecución de estudios explicativos más que descriptivos.

d) Evaluar los distintos Programas Pilotos desarrollados en torno a la juventud, en términos de su eficacia y eficiencia a fin de avanzar la operacionalización a nivel nacional de dichos Programas, preservando los factores socioculturales.

2. Difundir ampliamente los resultados de las investigaciones, Programas y diversas acciones que se ejecuten en cualesquiera de las áreas temáticas sobre la Juventud, con el objeto de que tanto los Ministerios como las I. Municipalidades y sus Corporaciones, los consideren en la elaboración de sus Políticas y/o Programas específicos. 
3. Friorizar la importancia del estudio de las aspiraciones y expectativas juveniles, bajo la forma de incluirlas como área temática específica de estudio de la Juventud, particularmente referido a las dimensiones de educación y trabajo.

4. Realizar Congresos Bienales de Investigadores sobre la Juventud Chilena, enfatizando la necesidad e importancia de estudios explicativos y evaluativos.

5. Llevar a cabo el II Congreso en el mes de junio de 1988.

6. Hacer llegar a las autoridades pertinentes las Conclusiones de este Congreso.

7. Editar un libro sobre la base de los trabajos aquí expuestos y de las conclusiones de cada Comisión Temática, que tendrá por objetivo sistematizar la información aquí resumida y generada a fin de difundirlo como primera aproximación a la temática de la Juventud.

\section{CONCLUSIONES TEMA: \\ DELINCUENCIA JUVENIL}

Moderador: Sra. Nina Horwitz $C$.

Secretaria: Srta. Isabel Ringelins $P$.

\section{CONCLUSIONES GENERALES}

1. Se refuerza la idea de la estrecha interdependencia entre la patología social y la patología psicológica frente al acto delictual, enfatizando algunos trabajos una perspectiva y otros, la otra.

2. Los hallazgos anteriores avalan intervenciones con una visión integral que abarque tanto la perspectiva psicológica, como la sociológica y judicial.

3. Se destaca la necesidad de continuar investigando la relación entre subcultura -comportamiento desviado y rehabilitación, en otras palabras, si el adolescente comete delitos que son funcionales y adoptativos para la subcultura inmediata pero disfuncionales para la sociedad... ¿Cómo enfrentar el problèma de la rehabilitación?

4. Se sugiere realizar estudios evaluativos que utilicen criterios sociológicos y psico-sociales para analizar el resultado de la rehabilitación del menor en situación irregular.

5. Sólo dos trabajos utilizan modelos teóricos, el modelo psicológico y el que se refiere a desviación primaria.

Se recomienda que en futuros trabajos se explicite el modelo de referencia que utiliza el autor o que de hecho se utilice un cuerpo teórico. Esto tendría a nuestro juicio dos ventajas: 
$1^{\circ}$ Enriquecería la interpretación de los resultados y sus implicaciones.

$2^{\circ}$ Fomentaría la revisión bibliográfica y teórica para el conocimiento de trabajos previos en el tema y que pueden servir de base al que se realiza.

\section{CONCLUSIONES COMISION TEMATICA:} JUVENTUD Y EXTREMA POBREZA

Moderador: Sr. Welnes Benjamín (FAO)

Secretaria: Srta. Teresa Figueras

- Los trabajos que se presentaron a esta comisión estaban orientados principalmente a interpretar la dinámica del comportamiento de los jóvenes a partir de conductas y expectativas específicas.

- Los trabajos teóricos que analizó esta comisión constituyen un real aporte y una nueva visión para el estudio empírico de la extrema pobreza y en la formulación de políticas sociales destinadas a satisfacer las necesidades básicas de los jóvenes de estos sectores.

- Se concluye a partir de los trabajos realizados que el nivel de expectativas laborales y profesionales de los jóvenes no está asociado de manera determinante al nivel socio-económico de éstos.

- Respecto de los jóvenes fumadores de marihuana de sectores de extrema pobreza se logró determinar que poseen una auto valoración positiva a pesar de su desmejorada situación, viéndose a sí mismo como un grupo "libre" y "amante de la paz".

Martes 17 de junio, 1986, 09:00 - 12:15 hrs.

Salón de Honor

Temática: SEXUALIDAD JUVENIL

Moderador: Sr. Luis Fuentealba Weber.

Secretario-Relator: Sr. Lautaro Arriagada Bravo.

La Comisión Temática que giró en torno a la "Sexualidad juvenil", examinó trece de los catorce trabajos presentados. En líneas generales cada uno de los ponentes expuso el resultado de sus investigaciones ciñéndose estrictamente a las versiones abreviadas enviadas al congreso y que fueron publicadas en el compendio repartido a cada una de las personas inscritas en el I Congreso Nacional de Investigadores Sociales y Médico-Sociales sobre la Juventud Chilena. 
A su vez y considerando la materia tratada en las investigaciones puede distinguirse:

1. Investigación de tipo sociológico.

2. Planificación familiar y oportunidades de Trabajo y Educación.

3. Investigación en torno al embarazo, situaciones de salud y patologías relacionadas con él.

El examen de los contenidos permite llegar a las siguientes conclusiones:

a) Hay una problemática sexual centrada en dos direcciones:

1) Carencia de una educación sexual adecuada que incluye como uno de sus temas principales la paternidad responsable. Pareciera que con ello se acentúa la afirmación de la O.M.S.: "La ignorancia es la causal de conductas negativas en el ser humano".

2) Se advierte una creciente proporción de embarazos en adolescentes especialmente en menores de 15 años con las consiguientes patologías y problemas psico-sociales.

b) La necesidad de la investigación permanente que se ciña estrictamente a los requerimientos de la metodología científica en lo conductual, biológico y socio-cultural tanto en la juventud masculina y femenina.

c) Se advierte la necesidad de una investigación nacional sobre sexualidad, a nivel de jóvenes de todas las edades, complementada con otra a nivel de adultos, llámense padres, apoderados, maestros, autoridades y otros.

d) En el aspecto metodológico relacionado con el estudio de las ponencias, se recomienda otra forma de trabajo, especialmente cuando hay gran número de trabajos como ocurrió en el caso particular de nuestra comisión en que predominó la temática "embarazo y sus diversos aspectos". Habría sido mejor realizar al respecto un panel que hubiese abarcado las variables consideradas por los investigadores o haber encargado a un expositor oficial el tema central y estudiar con subcomisiones la problemática central. 
CONCLUSIONES COMISION TEMATICA:

FAMILIA Y JUVENTUD

Moderador: Sra. Cristina Osorio

Secretaria: Sra. Inés Tardel

\section{CONCLUSIONES GENERALES}

1. Se confirmó una vez más la fuerza que tiene la familia como agente socializador para todos sus miembros.

2. Hay una aceptación mayoritaria de las normas sociales jurídicas y religiosas que regulan el matrimonio en nuestra sociedad.

3. La familia nuclear es la estructura claramente predominante en nuestra realidad.

4. La familia de procreación proyectada se enmarca dentro de las normas vigentes. Sólo difieren en cuanto a tamaño (menor número de hijos) y en cuanto a desempeño de roles, particularmente de pareja propendiendo a roles igualitarios intra y extra familiar.

5. La existencia de aproximadamente $1 / 5$ de familias nucleares incompletas, evidencian una socialización incompleta o sesgada en cuanto al rol de padre.

6. Se observan discrepancias entre los roles ideales y reales que cumplen los miembros del grupo familiar, particularmente el rol padre-abuelo y hermanos $y$ en menor grado el rol de la madre.

7. La valorización de la comunicación familiar es calificada mayoritariamente como buena.

8. El estudio de pautas culturales encontró que el nivel socio-económico alto, presenta pautas socializadoras que estimulan y/o refuerzan hacia mayor responsabilidad de los jóvenes.

9. Existe una clara diferencia entre los tres estratos estudiados en relación a los tópicos consultados, por lo que se recomienda programar de acuerdo a pautas culturales del estrato a los que se dirigen las medidas o contenido educativos, por lo tanto se propone que toda planificación educacional deje de ser estandarizada para todos, sin considerarse la variable socio cultural.

\section{RECOMENDACIONES}

Se sugiere tomar un catastro centralizado de los trabajos realizados en el país sobre familia y propender hacia la estandarización de instrumentos que faciliten posteriores estudios comparativos y concensales. 
COMISION TEMATICA:

JUVENTUD Y TRABAJO

Moderador: Sr. Oscar Corvalán Vásquez

Secretaria: Srta. Cecilia Jara Bernadot

\section{RESUMEN DE LAS EXPOSICIONES Y DISCUSION DE LAS MISMAS}

I. La presentación del trabajo "Educación y trabajo en jóvenes rurales de una Comuna de la zona Sur de Chile" estuvo a cargo del Dr. José Luis González, médico en Salud Pública y Licenciado en Educación. Este trabajo está referido a la situación de los jóvenes de la comuna de Los Lagos, ubicada en la X Región. Las conclusiones de esta investigación se pueden resumir en los siguientes puntos:

- Situación Demográfica: La comuna de Los Lagos es una de las comunas donde se dan las tasas más bajas de crecimiento de población. Esto se debe fundamentalmente a la gran cantidad de jóvenes que migran a ciudades cercanas (Valdivia, Osorno); el grupo etario entre 20 y 24 años es el que mira más, por esta razón en la investigación se consideró al grupo de jóvenes entre 15 y 19 años.

- Situación de Escolaridad de los jóvenes de Los Lagos:

a) Más de un $50 \%$ de los jóvenes no ha alcanzado más allá de sexto básico y la situación más deteriorada se presenta entre las mujeres.

b) Sólo un $5,4 \%$ alcanza al $3^{\circ}$ ó $4^{\circ}$ año de Enseñanza Media.

c) No más de un $20 \%$ ha tenido una transición regular por el sistema educativo; es decir, el $80 \%$ ha repetido uno o más cursos durante su permanencia en la escuela.

d) Cabe destacar que en esta zona existen posibilidades escolares hasta $6^{\circ}$ año básico; no existen posibilidades concretas para que los jóvenes rurales cursen el $8^{\circ}$ año de enseñanza básica, que se estima como obligatorio.

e) Existen altas tasas de deserción escolar; se pudo ver en la investigación que este fenómeno está asociado al costo que implica la Educación en relación al nivel de ingreso familiar.

f) La baja capacidad de retención del sistema escolar se expresa en el $10 \%$ de analfabetos por desuso, jóvenes que sólo cursaron el $1^{\mathrm{er}}, 2^{\circ} \mathrm{y}$ $3^{\text {er }}$ año básico. 
- Situación Laboral:

a) La proporción de jóvenes incorporados a la fuerza laboral en esta zona asciende a $69,6 \%$ cifra superior a la que considera la Encuesta Nacional de Empleo de diciembre de 1983, que consideraba a este grupo etario con un aporte de $18,5 \%$ para el país y un $31,7 \%$ para las zonas rurales.

b) La cifra de desocupación que muestra el INE para este grupo de la población es de $24,0 \%$ para el país y $12,4 \%$ para las zonas rurales; sin embargo, en esta comuna la tasa de desocupación asciende a un $62,6 \%$. Existe además un 18,3\% de jóvenes que trabajan sin percibir remuneraciones.

c) Los jóvenes aportan mano de obra barata, lo que reduce los costos de producción en esta zona que es básicamente forestal, razón por la cual aquí no existe trabajo temporal como sucede en muchas otras zonas rurales con trabajo agrícola.

A modo de conclusión se puede señalar que la educación y el trabajo son dos procesos básicos para la incorporación del joven a la sociedad, y viendo las cifras en relación a estos dos aspectos que presenta la Comuna de Los Lagos, se deduce que estos jóvenes se encuentran en una situación de extrema marginalidad.

II. La investigación referida a la "situación socio-laboral de la juventud chilena: diagnóstico y perspectivas" arrojó los siguientes datos que fueron presentados en este Congreso por los sociólogos Oscar Corvalán y Erika Santibáñez, investigadores del C.I.D.E. (Centro de Investigación y Desarrollo de la Educación).

- Magnitud y Caracteristicas de la Juventud: Hay que destacar que no existe una sola juventud, en este estudio se han considerado jóvenes a las personas que se encuentran entre 15 y 24 años. Esta es una definición eminentemente demográfica; sin embargo, la especificidad de este grupo está atravesada por factores de tipo geográfico (urbano, rural), económico y socio-culturales.

La proporción de los jóvenes del área urbana es cuatro veces mayor que la de los jóvenes de las áreas rurales y se distribuyen de manera similar entre hombres y mujeres. En el grupo de 20 a 24 años la participación laboral es del $92,1 \%$ en el área rural y de sólo un $52 \%$ en el área urbana. En tanto que en el área urbana son más los hombres que participan en la fuerza laboral que las mujeres; las mujeres de zonas rurales no reconocen su actividad doméstica como trabajo. La desocupación también afecta de manera diferencial a los jóvenes del campo y los de la ciudad, para el grupo adolescen- 
te (15 a 19) en 1982, 40\% de los jóvenes urbanos se encontraban desocupados frente a un $19 \%$ de los jóvenes rurales. Este fenómeno se repite para el grupo de 20 a 24 años. En las zonas urbanas no aparecen diferencias por sexo en las tasas de desocupación juvenil.

Las tasas de participación laboral de los jóvenes de estrato bajo son el doble de las que presentan los jóvenes de estrato socio-económico alto. EI crecimiento juvenil entre 1960 y 1985 casi se duplica en el grupo de jóvenes entre 15 a 24 años y el peso relativo de este grupo también aumentó del $17,9 \%$ a $22 \%$.

- Desempleo Juvenil: El desempleo juvenil no es parejo para los diferentes grupos socio-demográficos; los que se encuentran en situación de mayor desempleo son los hombres urbanos de 15 a 19 años y la menor tasa se presenta en jóvenes rurales de 20 a 24 años.

El desempleo juvenil está altamente correlacionado con las tasas de desempleo general, pero la desocupación golpea más fuertemente a los jóvenes de niveles socio-económicos bajos. Los jóvenes con mayor escolaridad presentan tasas de desocupación mayores, lo cual enfatiza la desvalorización económica de la educación.

Dado el crecimiento demográfico proyectado hasta el año 2.000 y el volumen actual de desempleo juvenil, se espera que para que disminuya sustantivamente la tasa de desempleo juvenil, el Producto Geográfico Bruto debería crecer a una tasa promedio anual superior al $4 \%$ en este período, con un crecimiento cero en el grupo de edad de jóvenes. De mantenerse las tasas históricas de crecimiento económico, el desempleo juvenil tendería a aumentar.

III. Proposiciones de la Comisión Juventud y Trabajo: A partir de la exposición del trabajo de los investigadores Dr. José Luis González y el Sr. Carlos Amtmann surgen las siguientes proposiciones en torno a la problemática Juventud $y$ Trabajo en el Area Rural.

1. Se hace necesario planificar políticas de desarrollo rural. No existe una coordinación del trabajo que realizan diversas instituciones en el área rural.

2. En cuanto a la educación se propone una educación centrada en el alumno, que rescate lo propio del campesino. Para esto se requiere de profesores comprometidos con el medio rural y dispuestos a insertarse en la comunidad ya que el trabajo en el campo no puede entenderse como una actividad individual.

3. En lo laboral se requiere de la creación de empleos productivos principalmente en la agroindustria, que satisfagan las necesidades de trabajo de la 
gran masa de desocupados que existen en el área rural ( $12 \%$ según cifras del INE), que en la Comuna de Los Lagos alcanza a un $62 \%$.

Tomando en consideración los datos expuestos por los sociólogos Oscar Corvalán y Erika Santibáñez en relación a la situación de los jóvenes chilenos surgen las siguientes proposiciones:

a) Se ha constatado que respecto de los jóvenes que desempeñan algún trabajo, en un $90 \%$ no utilizan los conocimientos que entrega la escuela. En este sentido, se propone un cambio curricular, de tal manera que efectivamente exista una educación para el trabajo.

b) Considerando, que el joven debe cambiar muchas veces de empleo y en otras pasar períodos de desempleo prolongado, se sugiere la creación de un sistema de educación profesional recurrente, que permita un reciclaje de los conocimientos del alumno, toda vez que los cambios tecnológicos así lo exigen.

c) Habiéndose declarado 1985 como el Año Internacional de la Juventud, se creó en nuestro país una Comisión Interministerial que se preocupara de conocer la situación de los jóvenes chilenos y proponer políticas tendientes a mejorar las condiciones económicas, psico-sociales, culturales y educativas. Al no existir un producto concreto de esta comisión se hace necesario estar constantemente levantando la problemática juvenil, para la creación e implementación de políticas juveniles efectivas, especialmente para dar respuesta al grave desempleo juvenil y sus consecuencias.

d) Se constata la carencia de un sistema educativo para jóvenes con problemas de aprendizaje. Los niños en la educación básica cuentan con la posibilidad de acceder a una educación básica diferencial; sin embargo, esta alternativa educativa se pierde en la enseñanza media. No se contempla la entrega de subvención para enseñanza media diferencial.

e) En estudios sobre características de los alumnos de escuelas técnicoprofesional queda demostrado que los niños más pobres son quienes participan de este sistema escolar. Los alumnos de sectores socioeconómicos bajos son quienes también tienen tasas más altas de deserción escolar antes del $10^{\circ}$ año de escolaridad, curso a partir del cual pueden acceder a un conocimiento respecto de un oficio. Tenemos entonces que los jóvenes de niveles más pobres no tienen acceso a una educación para el trabajo.

f) Por último, la Comisión recomienda la implementación de un verdadero sistema de educación permanente y formación profesional recurrente, a fin de que los individuos estén en condiciones de responder a los cambios del mundo del trabajo. 
COMISION TEMATICA:

ALCOHOLISMO Y DROGADICCION

Moderador: Dr. Ramón Florenzano

Secretaria: Dra. Virginia Canale

Se expusieron diez trabajos, los que pueden ser agrupados en tres áreas:

Los estudios confirmaron la alta frecuencia en el consumo de alcohol y marihuana y otras drogas entre los adolescentes y jóvenes.

Llamó la atención el consumo de solventes volátiles (Neoprén) entre los adolescentes de la región de Aysen; y las altas tasas de consumo de alcohol en las comunas de Santiago y Macul.

Se señaló también la importancia de los medios de comunicación en el fomento del consumo de alcohol. El uso inadecuado del tiempo libre y las variables macro-sociales en la génesis y mantención de los fármaco-dependientes.

Se subrayó la carencia de oportunidades laborales y la proyección a futuro de los jóvenes de hoy.

Conceptos clásicos como la anomia, alienación, síndrome amotivacional y otras innovativas como afinia, apropopositividad, se plantearon entre las posibles hipótesis explicativas.

Los factores de comercialización de substancias químicas (expendio de bebidas alcohólicas, redes de distribución clandestinas de alcohol y otros). Substancias que se situaron también entre los factores agravantes del problema.

La disonancia entre conocimientos y las actividades que existe entre las adolescentes. Esto se traduce en una incongruencia en la conducta real del individuo. Viéndose además la permisividad con que se visualiza el beber en el ámbito familiar del individuo.

\section{RECOMENDACIONES}

Es importante complementar los planes nacionales con las iniciativas locales y los que surgen del interés de la comunidad. De integrar la normativa del gobierno interior con la infraestructura y experiencia de los sistemas de salud, educación, justicia, etc...

Fomentar el desarrollo de acciones de prevención primaria a nivel de los sectores escolares y extraescolares.

Esto se relaciona con el mejor aprovechamiento del tiempo libre, con manejo publicitario adecuado, con mayor control por parte de las autoridades correspondientes en la comercialización de substancias químicas y alcohol. 
La importancia en el entrenamiento y fomento de Metodologías innovativas de investigación, por ejemplo los métodos utilizados en el estudio de la Comuna de Santiago, aproximaciones etnográficas e investigación, con la finalidad de trascender más allá de lo descriptivo, la búsqueda hacia factores explicativos de la problemática del adolescente actual.

\title{
CONCLUSIONES COMISION: JUVENTUD Y EDUCACION
}

\author{
Moderador: Sra. Iris Barrios \\ Secretario: Sr. Héctor Suárez
}

De los once trabajos expuestos en esta área temática, se desprende que la educación es un proceso realizado por personas y para personas. Es por lo tanto, un proceso eminentemente humano, que tiene como protagonista central al educando (niño, joven o adulto), siendo el resto de los participantes los profesores, padres y comunidad en general.

En los estudios presentados se analizaron diversos aspectos relativos a la inserción del joven en el sistema educativo formal.

Entre ellos, algunos se centran en la adaptación escolar, estudiando diversas variables sicológicas y psicosociales tales como: motivación, percepción de si mismo y del mundo, intereses, cogniciones, expectativas y aspiraciones; y otros, en la responsabilidad y actitudes de los agentes educativos (particularmente el profesor) en la promoción de esta adaptación.

Otros trabajos intentaron demostrar la relación entre las características socioeconómicas de los jóvenes y el tipo de establecimiento educacional al que asiste, dando como resultado una reproducción pedagógica de las condiciones de origen. Se concluye al respecto, que es equívoco hablar de un solo sistema escolar, en circunstancias que serían tres, que corresponderían a los establecimientos particulares pagados, municipales y particulares subvencionados, estableciéndose un paralelismo que describe distancias y oposiciones. La juventud, al pertenecer a distintas estructuras escolares, muestra orientaciones sociales diferentes, vivencias disímiles de sí y de su medio, proyectándose en forma distinta hacia el futuro.

Por otra parte, en relación a su autoimagen, los jóvenes evidencian un predominio del fatalismo y de la desvalorización personal. Esto se confirma en otro trabajo, que además detecta un grado importante de individualismo, apatía e inercia como expresiones juveniles frente a una sociedad que perciben amenazante. 
Respecto a la relación entre satisfacción de necesidades físicas, personales y sociales y el rendimiento académico, pareciera no existir una correlación significativa, siendo más bien el ambiente en el aula, las características familiares y el nivel socioeconómico los más determinantes. Otro estudio señala que los niveles reales de rendimiento académico son bajos en comparación a los objetivos propuestos en los programas de estudio de educación media, especialmente en relación con conocimientos matemáticos.

De los trabajos presentados se concluye finalmente, que el sistema de educación formal no estaría logrando los objetivos que se propone en cada nivel, tanto en lo relativo al rendimiento académico como a la adaptación psicosocial y a la orientación vocacional del joven.

En este sentido, pareciera existir una carencia importante de trabajos que vayan en la línea de proponer soluciones a estos problemas.

Creemos que este tipo de proposiciones, dada la complejidad del sistema educativo, debería surgir de estudios multidisciplinarios que aborden las distintas dimensiones que inciden en esta problemática.

CONCLUSIONES COMISION:

VALORES CULTURALES JUVENTUDES URBANA, RURAL Y MINORIAS ETNICAS

Moderador: Sr. Licer Viveros Cerda

Secretaria: Sra. María Cristina Di Silvestre

La primera conclusión se refiere a ratificar la validez del intervalo de edades entre 15 y 24 años como "edad juvenil" facilitando la investigación y la mantención de estándares internacionales de comunicación.

Las diferentes investigaciones expuestas sugieren, a pesar de las diferencias contextuales que la realidad psicosocial del joven chileno de hoy es más o menos uniforme, así lo demuestran las numerosas similitudes que aparecen en las diferentes áreas investigadas: urbana, semi urbana, rural y minorías étnicas.

Area Familiar: Reconocen sentirse amados por sus padres y que éstos han hecho mucho por ellos aun cuando al comparar a ambos padres, manifiestan que el padre es una figura lejana y es la madre la que juega un rol fundamental en su formación, es con ella con quien se comunican preferentemente, no obstante, sienten que sus padres no les brindan el apoyo suficiente, desean en ese sentido ampliar las oportunidades de comunicación y diálogo con el fin de que sus padres logren una mejor comprensión de la etapa que están viviendo. 
Area Educacional: Al respecto existen tres aspectos importantes de considerar:

1) Opinión acerca de los profesores

2) Forma y contenidos a ser estudiados

3) Objetivos de la educación.

1) Opinión acerca de los profesores: Los jóvenes dicen tener escasas relaciones con los profesores, no logran establecer con ellos relaciones personales más íntimas. La mayoría están muy lejanos del alumnado, sólo imparten sus materias, desplazándose de inmediato a otros lugares de trabajo.

2) Forma y contenidos a ser estudiados: Respecto de los programas de estudio, muchas de las materias no son del agrado de los jóvenes y creen que no les servirán en el futuro, consideran que tienen poco tiempo para estudiar respecto de la cantidad de asignaturas. Según ellos en la forma en que se imparten estas asignaturas subyacen los valores predominantes de la sociedad (rendimiento, competencia, éxito) sin dejar lugar para el desarrollo de la creatividad, la capacidad analítica y crítica.

3) Objetivos de la Educación: Para el joven el objetivo de la educación sería un medio para ingresar a la universidad y esta última un medio para conseguir trabajo. Al respecto se manifiesta una acentuada sobrevaloración de la universidad, a tal punto de generar un sentimiento de inseguridad e incertidumbre ante la posibilidad de no ingresar a ella.

Area Vocacional: Los jóvenes declaran que no existen posibilidades concretas de tipo ocupacional, expresan inseguridad e incertidumbre respecto a su futuro, a pesar de ello están muy preocupados por su realización personal y por el logro de un trabajo estable, una vez que obtengan su título. Sienten un gran vacío respecto de sus necesidades de orientación vocacional.

Area Relaciones de Amistad: Es altamente valorada y buscada por los jóvenes para compensar el sentimiento de soledad que experimentan al no lograr comunicación y comprensión que esperan de los adultos y muy especialmente de sus padres.

La amistad les permite tener un espacio para dialogar sobre su situación como jóvenes, desean compañía, comprensión y apoyo.

Sexualidad: Declaran haber iniciado tempranamente la actividad sexual, sin embargo, muestran insuficientes y equívocos conocimientos acerca de la sexualidad, especialmente en relación a las manifestaciones de su desarrollo sexual.

Demuestran desconocimiento y descuido en la prevención de los embarazos, persistiendo dudas y tabúes al respecto, asimismo una inconsistencia de sus juicios éticos en el ámbito sexual. 


\section{CONCLUSIONES COMISION:}

\section{SALUD MENTAL}

Moderador: Dr. Eduardo Medina C.

Secretario: Dr. Luis Caris N.

TEMAS ANALIZADOS EN LOS TRABAJOS PRESENTADOS

- Temas emocionales incorporados a la educación formal

- Trastornos emocionales asociados al embarazo precoz

- Suicidio

- Acciones de rehabilitación en deficientes mentales.

1. La salud mental es un campo muy amplio. Por sus características y problemáticas caben en ella numerosos aportes desde distintas perspectivas profesionales e interdisciplinarias.

2. Numerosas instituciones tienen vinculación en el campo, aunque con pocas posibilidades de investigar e intercambiar información. Estos Congresos son útiles para el intercambio de ellas.

A partir de ellos y las discusiones posteriores, se proponen las siguientes conclusiones:

3. La naturaleza de otros trabajos que fueron incluidos en otras Comisiones Temáticas también pertenecen al campo de la salud mental, por ejemplo, sexualidad, uso del tiempo libre, problemas de autoimagen, rendimiento académico estudiantil, violencia y delincuencia, alcoholismo y drogadicción, etc. En realidad, cualquier perspectiva que se tenga en la adolescencia y juventud tiene una estrecha vinculación con salud mental.

4. Los asistentes a la sala expresaron interés por los temas de los trabajos presentados y porque este conocimiento más las conclusiones de las comisiones temáticas sean llevados a las autoridades correspondientes como una contribución al abordaje de estos problemas.

5. A partir de opiniones técnicas, surgidas del campo médico asistencial, clínico y de salud pública, hubo consenso en la utilidad de las Ciencias Sociales para perfeccionar el estudio y enfrentamiento más eficaz de estos problemas.

6. Hubo numerosas opiniones en relación a que la Universidad de Chile diseñara mecanismos que faciliten el intercambio de experiencias de trabajo mediante reuniones de este tipo, tanto en Santiago como en otras Regiones y que contemplen la participación de interesados a lo largo del país. 
CONCLUSIONES COMISION TEMATICA:

JUVENTUD Y SALUD

Moderadora: Dra. Ana María Kaempfer $R$.

Secretaria: E.U. Edita Peña D.

La mayor parte de los trabajos presentados fueron de tipo descriptivo, aunque algunos podrían haber llegado a un nivel de análisis más profundo.

Hubo un conjunto de cinco trabajos relacionados con diversas formas de atención del adolescente, especialmente en centros especializados.

La característica más común de estos centros fue de trabajar con adolescentes en algún nivel de escolaridad y referido por profesores, a excepción de uno de ellos como es el caso de estudiantes de nivel superior que tenían un servicio médico recientemente organizado para acceso libre, no referido.

En general, los diferentes autores recalcan la gran variedad de problemas que presenta este grupo de edad, que incluyó desde egresados de la enseñanza básica hasta estudiantes universitarios.

Los trabajos presentados mostraron una variedad de formas de recolección de información donde la información directa dada por el propio adolescente ocupó un lugar destacado y que el grupo que he referido o que consulta, es sólo una fracción del universo estudiado. Esto último puede ser interpretado por una parte por la escasez de recursos que tradicionalmente se han destinado a atender al grupo de adolescentes mayores ( 15 a 19 y 20 a 24 años) y por otra parte, que efectivamente son pocos quienes la necesitan.

Otra constante observada en los trabajos presentados es la de que, pese a la creencia común que se tiene de que los adolescentes presentan problemas de preferencia referidos al área de salud mental, al analizar los perfiles de morbilidad se aprecia una gama amplia de problemas de salud física.

Como consecuencia de la situación observada, los autores en forma más o menos unánime reconocen la necesidad de equipos multidisciplinarios, o, por lo menos, acceso fácil a las diferentes especialidades que la atención integral requiere.

Por otra parte, se nota claramente en los trabajos y se confirmó en las presentaciones que la atención del adolescente debe tener una calidad humana de más confianza, intimidad e independencia de parte del adolescente consultante.

El análisis de un aspecto específico de la nutrición de los adolescentes demostró una serie de defectos, entre los que destaca como preocupante el exceso de consumo proteico y de colesterol y los déficits importantes y generalizados de vitamina $A$ en ambos sexos y de fierro en las niñas. 
La obesidad parece ser un problema de mayor importancia que la desnutrición y en ese sentido resultó novedosa y prometedora la presentación sobre un Programa de acondicionamiento físico con metodología de cohorte antes y después, realizado en la Universidad del Norte.

Como situaciones particulares de la problemática de salud del adolescente se abordaron los temas referentes a muertes violentas y embarazo precoz en la adolescente menor de 16 años de edad y su trascendencia social y psicológica.

Con respecto a las violencias se concluye que éstas constituyen la primera causa de muerte en los jóvenes, que ésta es mayor a mayor edad del adolescente y que es el adolescente hombre el que tiene mayor riesgo. Por otra parte se observa que a igual que el adulto, el adolescente se ve afectado por factores sociales, además de los psicológicos y familiares de la edad, en este aspecto de las muertes violentas.

Con respecto al embarazo precoz, estudio multidisciplinario de la VIII Región, se puede concluir que en general es un embarazo no deseado, pero que, producido el hecho, la mayor aceptación se concentra en la familia materna y ésta pasa a tener un rol importante en la situación futura de la adolescente gestante y de su hijo. Por otra parte la actitud más frecuente observada en el progenitor es la de abandono y no compromiso, lo que a juicio de los autores se debe más bien a la situación socio-económica por ser un progenitor también adolescente. Otro aspecto importante que se observó fue que mientras la familia de origen tiende hacia la legitimidad, la familia de procreación tiende a la ilegitimidad, situación que debe ser motivo de preocupación por las consecuencias sociales y psicológicas tanto para el niño como para su futura familia.

Por último, se presentó un trabajo relacionado con el grado de conocimientos y actitudes referidos a sexualidad y reproducción humana, donde se pudo una vez más observar el alto grado de ignorancia en que se encuentran los adolescentes especialmente en lo referente a sexualidad humana, haciéndose esto más agudo en zonas rurales de nuestro país. 